\title{
A!
}

This is an electronic reprint of the original article.

This reprint may differ from the original in pagination and typographic detail.

Chen, Xi Rong; Chen, Guangze; Zheng, Yue; Chen, Wei; Xing, D. Y.

\section{Conductance oscillation in surface junctions of Weyl semimetals}

Published in:

Physical Review B

DOI:

10.1103/PhysRevB.104.205412

Published: 08/11/2021

Document Version

Publisher's PDF, also known as Version of record

Please cite the original version:

Chen, X. R., Chen, G., Zheng, Y., Chen, W., \& Xing, D. Y. (2021). Conductance oscillation in surface junctions of Weyl semimetals. Physical Review B, 104(20), [205412]. https://doi.org/10.1103/PhysRevB.104.205412

This material is protected by copyright and other intellectual property rights, and duplication or sale of all or part of any of the repository collections is not permitted, except that material may be duplicated by you for your research use or educational purposes in electronic or print form. You must obtain permission for any other use. Electronic or print copies may not be offered, whether for sale or otherwise to anyone who is not an authorised user. 


\title{
Conductance oscillation in surface junctions of Weyl semimetals
}

\author{
Xi-Rong Chen, ${ }^{1,2, *}$ Guangze Chen $\odot,{ }^{3, *}$ Yue Zheng, ${ }^{1,2}$ Wei Chen, ${ }^{1,2, \dagger}$ and D. Y. Xing ${ }^{1,2}$ \\ ${ }^{1}$ National Laboratory of Solid State Microstructures and department of Physics, Nanjing University, Nanjing 210093, China \\ ${ }^{2}$ Collaborative Innovation Center of Advanced Microstructures, Nanjing University, Nanjing 210093, China \\ ${ }^{3}$ Department of Applied Physics, Aalto University, 02150, Espoo, Finland
}

(Received 26 July 2021; revised 12 October 2021; accepted 27 October 2021; published 8 November 2021)

\begin{abstract}
Fermi arc surface states, the manifestation of the bulk-edge correspondence in Weyl semimetals, have attracted much research interest. In contrast to the conventional Fermi loop, the disconnected Fermi arcs provide an exotic two-dimensional (2D) system for exploration of novel physical effects on the surface of Weyl semimetals. Here, we propose that visible conductance oscillation can be achieved in planar junctions fabricated on the surface of a Weyl semimetal with a pair of Fermi arcs. It is shown that Fabry-Pérot-type interference inside the 2D junction can generate conductance oscillation with its visibility strongly relying on the shape of the Fermi arcs and their orientation relative to the strip electrodes, the latter clearly revealing the anisotropy of the Fermi arcs. Moreover, we show that the visibility of the oscillating pattern can be significantly enhanced by a magnetic field perpendicular to the surface taking advantage of the bulk-surface connected Weyl orbits. Our work offers an effective way to identify Fermi arc surface states through transport measurement and predicts the surface of Weyl semimetals as a novel platform for the implementation of 2D conductance oscillations.
\end{abstract}

DOI: 10.1103/PhysRevB.104.205412

\section{INTRODUCTION}

The Weyl fermion is a massless fermionic particle with definite chirality named after Hermann Weyl [1], and was proposed originally as a candidate for fundamental particles. Though it plays an important role in quantum theory and the standard model, the verification of Weyl fermions in high-energy physics remains elusive [2-4]. Recently, the Weyl fermion has been observed unexpectedly in an alternative form as quasiparticle excitations in a class of condensed matter materials called Weyl semimetals (WSMs) [5], thereby inspiring research activities on Weyl physics and opening a new avenue for exploration of relativistic Weyl fermions in solid-state physics [6-21]. In contrast to its high-energy counterpart, the exotic properties of Weyl fermions in solid-state physics are usually manifested as anomalous transport and optical phenomena [22-36].

One unique feature of the WSMs is the existence of Fermi arc (FA) surface states at its boundaries [5], without any highenergy counterpart. According to the no-go theorem [37,38], the Weyl points in a WSM must appear in pairs with opposite chirality [39], with FA spanning between each pair in the surface Brillouin zone [5]. Such disconnected FAs are the fingerprint of WSMs [9-21], which play a key role in the identification of WSMs in experiments [10-20]. Most experiments on Weyl semimetals focus on the angle-resolved photoemission spectroscopy, in which the existence of FAs has been confirmed. There are also theoretical studies of the FA and its transport properties [40-44], like quasiparticle interference (QPI) $[45,46]$. Recent progress has also shown

\footnotetext{
*These authors contributed equally to this work.

$\dagger$ Corresponding author: pchenweis@gmail.com
}

that the configurations of the FAs are sensitive to the details of the sample boundary [47-49], thus opening the possibility for engineering FAs and exploring their novel effects and potential applications through surface device fabrication and transport measurements. In contrast to the photoemission spectroscopy experiments, the surface transport measurement has the advantage of extracting useful information of the spatial distribution of the surface states [50-52].

In this work, we propose that novel 2D conductance oscillation stemming from Fabry-Pérot-type interference can be realized in the planar normal metal-FA-normal metal (NFAN) junctions on the surface of the WSM. The junctions consist of two strips of normal metal electrodes mediated by a pair of FA surface states in between as shown in Figs. 1(a) and 1(b). Our main findings in this work are that (i) shorter and less curved FAs can lead to more visible conductance oscillation stemming from a weaker dephasing effect between different transverse channels, (ii) the oscillation pattern of the conductance strongly relies on the relative orientation between the FAs and the strip electrodes denoted by the azimuthal angle $\theta$ in Fig. 1(b), and (iii) the visibility of the conductance oscillation can be significantly enhanced by a magnetic field perpendicular to the planar junctions due to the existence of the magnetic Weyl orbit. Our work shows that FA surface states offer a novel platform to observe 2D conductance oscillation in addition to the existing systems such as graphene [53-58] and the inverted InAs/GaSb double quantum well [59]. The orientation dependence and the field modulation of the conductance provide a unique signature of the FAs, which can be used for identifying WSMs through a transport approach.

The rest of this paper is organized as follows: In Sec. II, we present effective models for a time-reversal $(\mathcal{T})$ symmetric WSM and its FA surface states. We then show that a general 
(a)

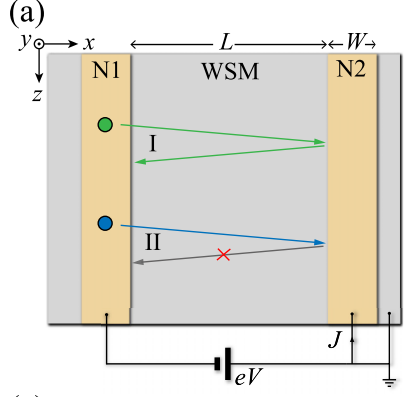

(c)

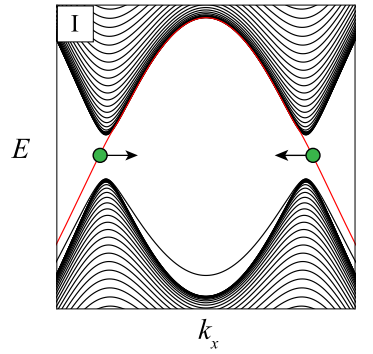

(b)

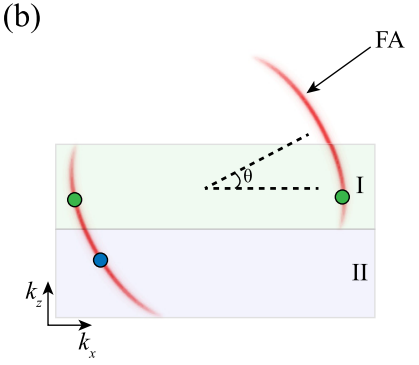

(d)

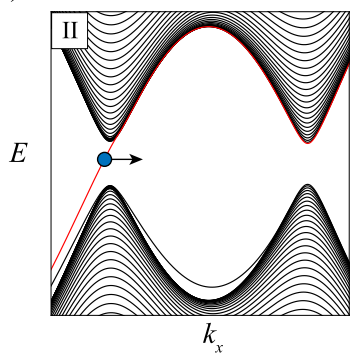

FIG. 1. (a) Schematic illustration of the planar normal metal (N)-FA-normal metal $(\mathrm{N})$ on the top surface of a WSM slab and scattering of particles at the interface. The trajectories of electrons (solid circles) are sketched as solid lines, where the colors of them denote that they belong to different $k_{z}$ regions. (b) Two regions I and II for left incident electrons are defined by the transverse momentum $k_{z}$. (c) The corresponding band structures for a fixed $k_{z}$ of the two regions. The red lines denote the FA top surface states and black arrows indicate the moving directions.

oriented FA can be described by applying a rotation transformation of the effective Hamiltonian. Based on the effective models and using the Green's function approach, we show analytically the existence of oscillations in the conductance spectra of a NFAN junctions on the WSM surface in Sec. III and support the analytical results with numerical simulations on the lattice model. In Sec. IV, we show the dependence of such oscillation on the relative orientation of the FAs to the normal metals with numerical means. In Sec. V, we show that the oscillation can be enhanced by applying a magnetic field perpendicular to the WSM surface. Finally, we give a brief summary in Sec. VI.

\section{II. $\mathcal{T}$-SYMMETRIC WEYL SEMIMETAL AND FA SURFACE STATES}

We adopt the following effective two-band $k \cdot p$ model, which describes a $\mathcal{T}$-symmetric Weyl semimetal with four Weyl points [51,52]:

$$
H_{W}^{0}(\boldsymbol{k})=M_{1}\left(k_{1}^{2}-k_{x}^{2}\right) \sigma_{x}+v_{y} k_{y} \sigma_{y}+M_{2}\left(k_{0}^{2}-k_{y}^{2}-k_{z}^{2}\right) \sigma_{z},
$$

where $v_{y}$ is the velocity in the $\hat{y}$ direction, $M_{1,2}$ and $k_{0,1}$ are model parameters, and $\sigma_{x, y, z}$ are the Pauli matrices in the pseudospin space. The valence and conduction bands cross linearly at four Weyl points $\boldsymbol{k}_{W}=\left( \pm k_{1}, 0, \pm k_{0}\right)$. The low-energy Hamiltonian near the Weyl points are $h_{W}(\boldsymbol{k})=$ $\pm 2 M_{1} k_{1} k_{x} \sigma_{x}+v_{y} k_{y} \sigma_{y} \pm 2 M_{2} k_{0} k_{z} \sigma_{z}$. We are interested in the topologically protected FA surface states on the open surface

in the $\hat{y}$ direction. They are confined by $\left|k_{z}\right|<k_{0}$ and can be described by

$$
H_{\text {Arc }}^{0}\left(k_{x}\right)=M_{1}\left(k_{x}^{2}-k_{1}^{2}\right),
$$

with two straight FAs defined by $k_{x}= \pm k_{1}$. Generically, FAs in real materials are curved, so we introduce a dispersion term $\epsilon\left(k_{z}\right)=d\left(k_{z}^{2}-k_{0}^{2}\right)$ to capture that feature; the total Hamiltonian of the surface states is

$$
H_{\text {Arc }}(\boldsymbol{k})=H_{\text {Arc }}^{0}\left(k_{x}\right)+\epsilon\left(k_{z}\right),
$$

with the in-plane wave vector $\boldsymbol{k}=\left(k_{x}, k_{z}\right)$.

One important feature of the FAs is strong anisotropy. In the planar junctions, the relative orientation between the FAs and the normal of the strip electrodes denoted by the angle $\theta$ [Fig. 1(b)] strongly affects the physical results. In the long wavelength limit, we can apply a rotational transformation to the effective Hamiltonian Eq. (1) of WSM to describe such an effect while fixing the direction of the electrodes at the same time. A rotation about the $\hat{y}$ axis by an angle $\theta$ is described by

$$
H_{W}(\boldsymbol{k}, \theta)=H_{W}^{0}\left(U_{y}^{-1} \boldsymbol{k}\right)
$$

with the rotation operator

$$
U_{y}(\theta)=\left(\begin{array}{ccc}
\cos \theta & 0 & \sin \theta \\
0 & 1 & 0 \\
-\sin \theta & 0 & \cos \theta
\end{array}\right) .
$$

The locations of Weyl points determined by $H_{W}(\boldsymbol{k}, \theta)=0$ are transferred to $U_{y}(\theta) \boldsymbol{k}_{W}= \pm\left(k_{1} \cos \theta+\right.$ $\left.k_{0} \sin \theta, 0,-k_{1} \sin \theta+k_{0} \cos \theta\right)$ and the FAs terminated at these points rotate accordingly [cf. Fig. 1(b)]. In the next section, we show the conductance oscillation in the planar junctions with the dispersion (3), and in Sec. IV we show the dependence of such oscillation on the orientation of the FAs based on the discrete version of Hamiltonian (4).

\section{CONDUCTANCE OSCILLATION IN NFAN JUNCTIONS}

The WSM surface with FA states is a novel 2D system, which differs from other systems with closed Fermi loops. The disconnected nature of FA may lead to the absence of backscattering channels in surface transport [52]. In particular, consider the NFAN junctions as shown in Fig. 1(a) with the FA having an azimuthal angle $\theta$ relative to the normal metal electrodes [Fig. 1(b)]. In region I of the surface Brillouin zone, there exists two counter-propagating channels at the Fermi surface [Fig. 1(c)], thus enabling back-scattering. In contrast, in region II, there exists a single chiral channel [Fig. 1(d)], and back-scattering is prohibited. Interestingly, the ratio between regions I and II depends solely on the relative orientation $\theta$. We show first the existence of conductance oscillation with $\theta=0$ in this section, and investigate its $\theta$ dependence in the next section.

\section{A. Analytical calculation}

We investigate the ballistic transport in the NFAN junctions using the Green's function method. We consider the case $\theta=0$ first, where all conducting channels are of type I in the surface Brillouin zone (cf. Fig. 1). The surface Hamiltonian is 
captured by $H_{\text {Arc }}(\boldsymbol{k})$ in Eq. (3). The tunneling Hamiltonian

$$
H_{T}=\sum_{p, \alpha=1,2} T_{\alpha} d_{p, \alpha}^{\dagger} \psi\left(x_{\alpha}\right)+\text { H.c. }
$$

is adopted to describe the coupling between the FA surface states and the normal electrodes where $T_{\alpha}$ is the tunneling strength between the surface states and the $\alpha$ electrode [cf. Fig. 1(a)], $d_{p, \alpha}$ is the Fermi operator in the $\alpha$ terminal with momentum $p$, and $\psi\left(x_{\alpha}\right)$ is the field operator of the surface states at each terminal located at $x_{\alpha}$. Here we adopt a constant tunneling strength for simplicity to catch the main physics. A momentum-dependent tunneling can only lead to certain quantitative correction to the conductance spectra without changing the configuration of the oscillation pattern.

For the planar junctions with good quality of the strip electrodes, the transverse momentum $k_{z}$ is approximately conserved during scattering. The differential conductance (without spin degeneracy) per unit length of the strip electrodes is the summation over transmissions in all $k_{z}$ channels as

$$
\sigma(\varepsilon)=\frac{e^{2}}{h} \int_{-k_{0}}^{k_{0}} \widetilde{T}\left(\varepsilon, k_{z}\right) d k_{z},
$$

with $\varepsilon$ being the electron energy. The range of integration is limited by the spreading of FAs in the $k_{z}$ direction. The $k_{z^{-}}$ dependent transmission function $\widetilde{T}\left(\varepsilon, k_{z}\right)$ can be calculated by the nonequilibrium Green's function method through [60]

$$
\widetilde{T}\left(\varepsilon, k_{z}\right)=\operatorname{Tr}\left[\Gamma_{1} G^{R} \Gamma_{2} G^{A}\right],
$$

where $\Gamma_{1,2}$ are linewidth functions of the leads and $G^{R, A}$ are the full retarded and advanced Green's functions, respectively. For a given energy $\varepsilon$ and transverse momentum $k_{z}$, there are two counterpropagating channels with momenta $\pm k_{x}^{0}$ and $k_{x}^{0}\left(\varepsilon, k_{z}\right)=\sqrt{k_{1}^{2}+\frac{\varepsilon-d\left(k_{z}^{2}-k_{0}^{2}\right)}{M_{1}}}$. The bare Green's functions can be obtained as

$$
\begin{aligned}
g_{\varepsilon}^{R}\left(x^{\prime}, x\right) & =\left[g_{\varepsilon, k_{z}}^{A}\left(x, x^{\prime}\right)\right]^{*}=g_{\varepsilon, k_{z}}^{R}\left(x, x^{\prime}\right) \\
& =-\pi i \rho_{S}\left(\varepsilon, k_{z}\right) e^{i k_{0}\left(x^{\prime}-x\right)},
\end{aligned}
$$

with $\rho_{S}=\frac{1}{4 \pi M_{1} k_{x}^{0}}$. The full Green's function and the linewidth function can be calculated in the standard way by taking into account the tunneling term $H_{T}$, which gives

$$
\begin{aligned}
G_{\varepsilon}^{R}\left(x_{2}, x_{1}\right) & =\frac{g_{\varepsilon}^{R}\left(x_{2}, x_{1}\right)}{\left(1+R_{1}\right)\left(1+R_{2}\right)-R_{1} R_{2} f_{\varepsilon}\left(x_{2}, x_{1}\right)}, \\
\Gamma_{\alpha}\left(x, x^{\prime}, \varepsilon\right) & =2 \pi \rho_{\alpha}(\varepsilon)\left|T_{\alpha}\right|^{2} \delta\left(x-x_{\alpha}\right) \delta\left(x^{\prime}-x_{\alpha}\right) .
\end{aligned}
$$

where $f_{\varepsilon}\left(x_{2}, x_{1}\right)=e^{2 i k_{0}\left(x_{2}-x_{1}\right)}, R_{\alpha}(\varepsilon)=\pi^{2} \rho_{S}(\varepsilon) \rho_{\alpha}(\varepsilon)\left|T_{\alpha}\right|^{2}$, and $\rho_{\alpha}$ is the density of states of the leads. We have assumed that $T_{\alpha}$ and $\rho_{\alpha}$ are $k_{z}$ independent such that $\Gamma_{\alpha}$ has no $k_{z}$ dependence. We have also neglected the $k_{z}$ dependence of $\rho_{S}$ that does not qualitatively change the result. The transmission coefficient in Eq. (8) reduces to

$$
\widetilde{T}\left(\varepsilon, k_{z}\right)=\frac{4 R_{1} R_{2}}{\left|\left(1+R_{1}\right)\left(1+R_{2}\right)-R_{1} R_{2} f_{\varepsilon}\left(x_{2}, x_{1}\right)\right|^{2}} .
$$

The Fabry-Pérot-type interference is indicated by the coherence factor $f_{\varepsilon}\left(x_{2}, x_{1}\right)$ in the transmission function, which induces the oscillation of $\widetilde{T}$ with varying $\varepsilon$. It also exhibits a $k_{z}$ dependence, meaning that different transverse channels can have a relative phase shift; see Fig. 2. From the expression of the conductance (7), one can infer that a strong dephasing between the $k_{z}$ channels will suppress the overall oscillation of the conductance by phase averaging. This is the main reason why the Fabry-Pérot oscillation of the conductance in a 2D metal is hard to implement [59]. In contrast to a closed Fermi surface, the terminated FAs can effectively reduce the dephasing effect, so that the FA surface states provide a promising 2D platform to implement conductance oscillation. From the physical picture above, we can infer that FAs with smaller curvature and shorter length result in more visible oscillation, which is verified in Fig. 2.

\section{B. Numerical simulation}

Next, we perform numerical simulation of the conductance oscillation on the lattice model. Assuming that the size of both strip electrodes in the $\hat{z}$ direction is much larger than the Fermi wavelength and their boundaries are smooth enough, then the transverse momentum $k_{z}$ is approximately conserved during scattering and can be regarded as a parameter. In this way, the numerical calculation is reduced to a set of $2 \mathrm{D}$ slices labeled by $k_{z}$. For $\left|k_{z}\right|<k_{0}$, a pair of edge states emerge under the open boundary condition [Fig. 1(c)]. By the substitutions $k_{i=x, y, z} \rightarrow \frac{1}{a} \sin k_{i} a$ and $k_{i}^{2} \rightarrow \frac{2}{a^{2}}\left(1-\cos k_{i} a\right)$ while keeping $k_{z}$ as a parameter, we obtain the lattice version of the Hamiltonian (1) as

$$
\begin{aligned}
\mathcal{H}_{W}\left(k_{z}\right)= & \sum_{i} c_{i}^{\dagger} H_{i i} c_{i}+\sum_{i}\left(c_{i}^{\dagger} H_{i, i+a_{x}} c_{i+a_{x}}+\text { H.c. }\right) \\
& +\sum_{i}\left(c_{i}^{\dagger} H_{i, i+a_{y}} c_{i+a_{y}}+\text { H.c. }\right)
\end{aligned}
$$

where $c_{i}=\left(c_{i, \uparrow}, c_{i, \downarrow}\right)$ is the Fermi operator on site $i=\left(i_{x}, i_{y}\right)$ with two pseudospin components, $a_{x}=(a, 0)$ and $a_{y}=(0, a)$ are the unit vectors along the $\hat{x}$ and $\hat{y}$ directions, respectively, with $a$ being the lattice constant. $H_{i i}$ and $H_{i, i+a_{x}\left(a_{y}\right)}$ are $2 \times 2$ block matrices and take the explicit forms as

$$
\begin{aligned}
H_{i i} & =M_{1}\left(k_{1}^{2}-\frac{2}{a^{2}}\right) \sigma_{x}+M_{2}\left(k_{0}^{2}-\frac{4}{a^{2}}+\frac{2}{a^{2}} \cos k_{z} a\right) \sigma_{z}, \\
H_{i, i+a_{x}} & =\frac{M_{1}}{a^{2}} \sigma_{x}, \quad H_{i, i+a_{y}}=\frac{v_{y} i}{2 a} \sigma_{y}+\frac{M_{2}}{a^{2}} \sigma_{z} .
\end{aligned}
$$

The configuration of FAs on the top surface can be revealed by the spectra function $\mathcal{A}(\varepsilon)=-\frac{1}{\pi} \operatorname{Im} g_{W}^{R}(\varepsilon)$ in the top layer at $\varepsilon=0$, with $g_{W}^{R}\left(\varepsilon, k_{x}, k_{z}\right)$ being the retarded Green's function calculated by the lattice model of the Weyl semimetal under open boundary condition in the $\hat{y}$ direction; see Fig. 3(a). To simulate the FAs in real materials [49,61-63], we have introduced an on-site potential $V$ on the top layer of the WSM lattice to introduce surface dispersion that yields curved FAs [51,52].

The strip electrodes can be described by an effective Hamiltonian $H_{N}(\boldsymbol{k})=\left(C \boldsymbol{k}^{2}-\mu_{N}\right) \sigma_{0}$, with $C$ being the parameter related to the effective mass, $\mu_{N}$ being the chemical potential, and $\sigma_{0}$ being the identity matrix. The lattice model 
(a)

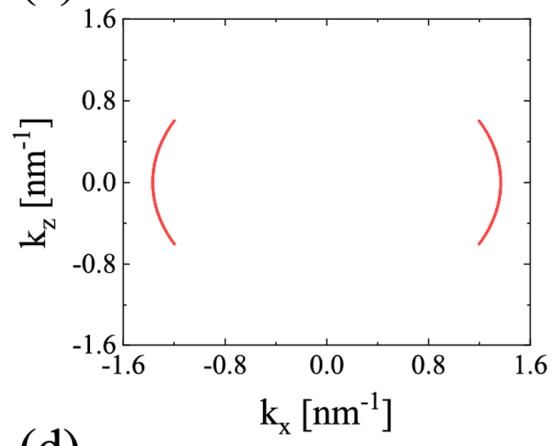

(d)

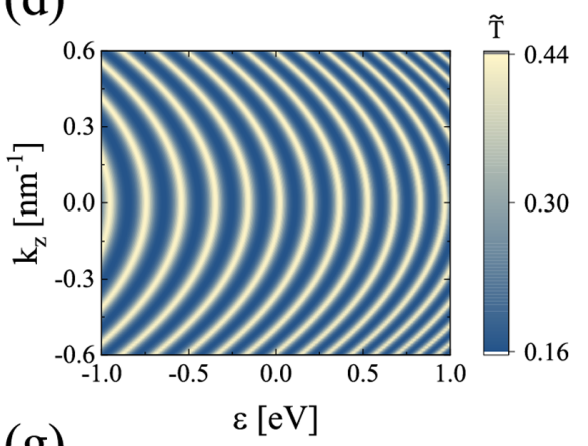

(g)

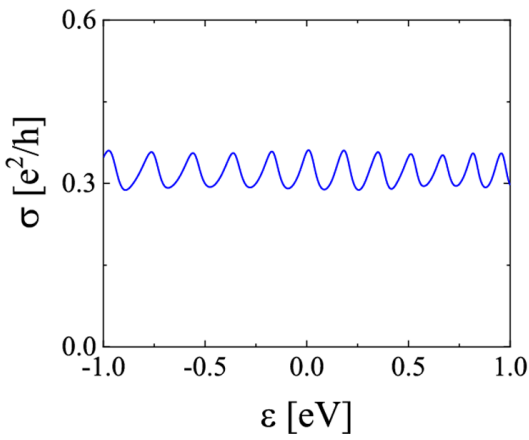

(b)

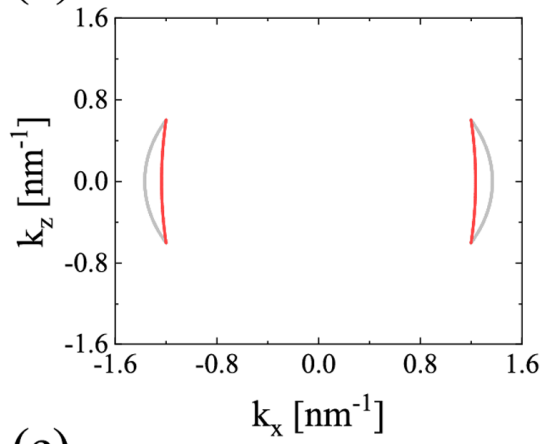

(e)

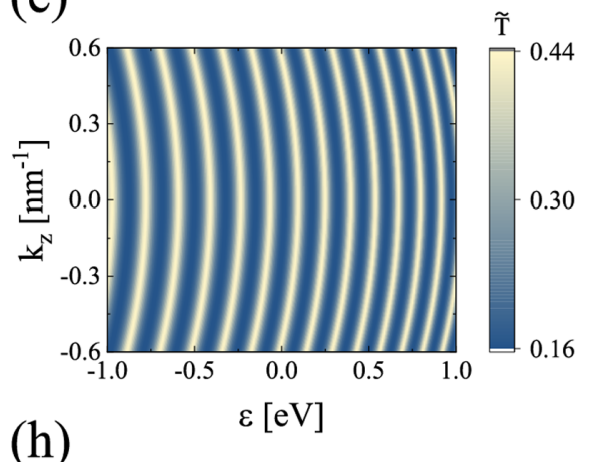

(h)

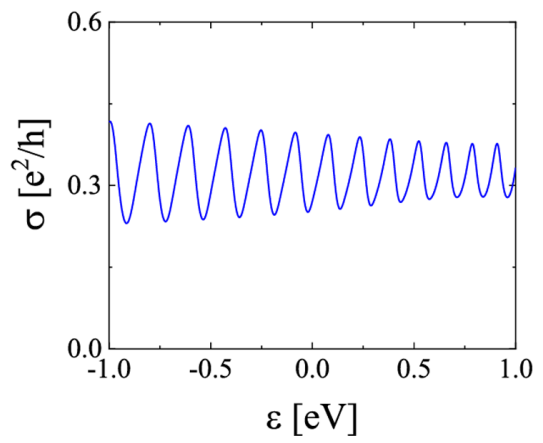

(c)

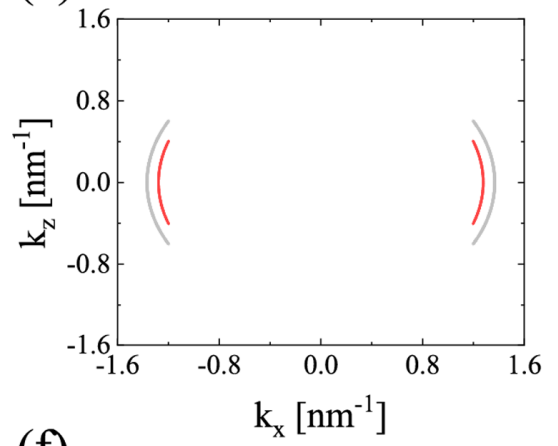

(f)

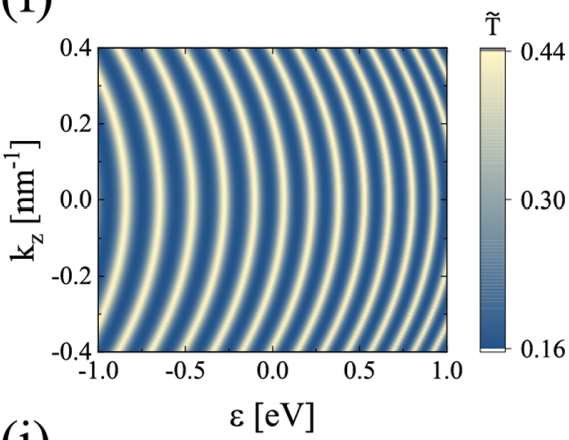

(i)

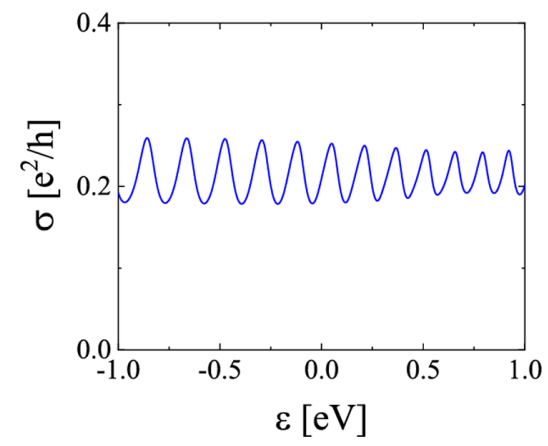

FIG. 2. Conductance oscillations for different FAs given by Eq. (3). Top rows illustrate the FAs with different curvature and length, where the relevant parameters are (a) $k_{0}=0.6 \mathrm{~nm}^{-1}, d=1.5 \mathrm{eV} \mathrm{nm}^{2}$; (b) $k_{0}=0.6 \mathrm{~nm}^{-1}, d=0.3 \mathrm{eV} \mathrm{nm}^{2}$; and (c) $k_{0}=0.4 \mathrm{~nm}^{-1}, d=1.5 \mathrm{eV} \mathrm{nm}^{2}$. [(d)-(f)] Middle rows illustrate the corresponding analytical results of the transmission coefficient $\widetilde{T}(\theta=0)$ as a function of $\varepsilon$ and $k_{z}$. [(g)-(i)] Bottom rows exhibit the corresponding conductance $\sigma$ after integrates over $k_{z}$. The other parameters are set as $a=1 \mathrm{~nm}, R_{1}=R_{2}=1$, $M_{1}=1.25 \mathrm{eV} \mathrm{nm}^{2}$, and $k_{1}=1.2 \mathrm{~nm}^{-1}$.

for the electrodes can be obtained in a similar way as

$$
\mathcal{H}_{N}\left(k_{z}\right)=\sum_{j} d_{j}^{\dagger} \lambda_{j} d_{j}-\frac{C}{a^{2}} \sum_{j}\left(d_{j}^{\dagger} d_{j+a_{x}}+d_{j}^{\dagger} d_{j+a_{y}}+\text { H.c. }\right),
$$

where $d_{j}=\left(d_{j, \uparrow}, d_{j, \downarrow}\right)$ is the Fermi operator, and $\lambda_{j}=$ $2 C\left(3-\cos k_{z} a\right) / a^{2}-\mu_{N}$.

The whole system for a given $k_{z}$ is described by $\mathcal{H}_{W}\left(k_{z}\right)$ and $\mathcal{H}_{N}\left(k_{z}\right)$ and the coupling between them which is captured by the tunneling between the outmost lattice layers with a strength $t_{N}$. The thicknesses of the WSM and the electrodes in the $\hat{y}$ direction are 100 and $40 \mathrm{~nm}$, respectively. The width of the hopping area in the $\hat{x}$ direction is $W=30 \mathrm{~nm}$, and the separation between two electrodes is $L=180 \mathrm{~nm}$ [cf. Fig. 1(a)]. Two on-site potentials $U_{1}$ and $U_{2}$ are introduced at the boundary of the $N$ electrodes to simulate the interface barrier or the momentum mismatch in the heterostructure Both the WSM and electrodes connect to the leads extended to infinity in the $\pm \hat{x}$ directions. The transmission $T\left(k_{z}, \varepsilon\right)$ between two electrodes is calculated using the KWANT program [64]. The overall conductance by summing up all the transverse channels can be obtained as

$$
\sigma(\varepsilon)=\frac{e^{2}}{h} \int_{-k_{0}}^{k_{0}} T\left(k_{z}, \varepsilon\right) d k_{z}
$$

In the case of $\theta=0$, the FAs with different curvature and length are shown in Figs. 3(a)-3(c). The corresponding results of the transmission probability $T\left(k_{z}, \varepsilon\right)$ and the differential conductance $\sigma(\varepsilon)$ are plotted in Figs. 3(d)-3(f) and Figs. 3(g) $3(\mathrm{i})$, respectively. One can see from Fig. 3 that less curved and shorter FAs result in more visible conductance oscillation, in which the dephasing effect between transverse channels becomes weaker as revealed by the length and curvature of 
(a)
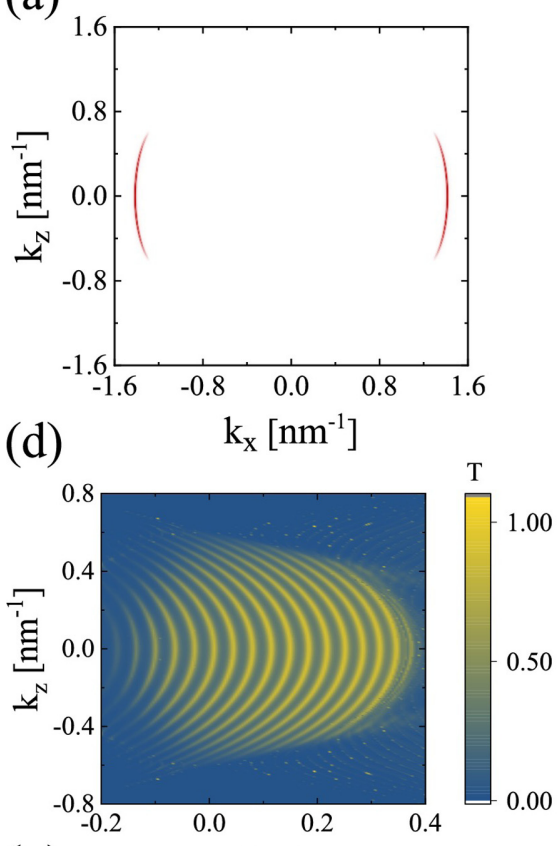

(g)

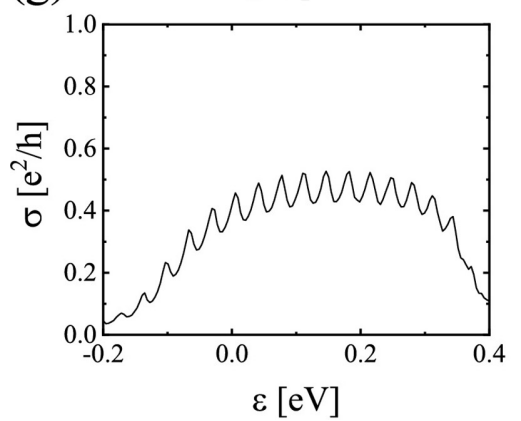

(b)
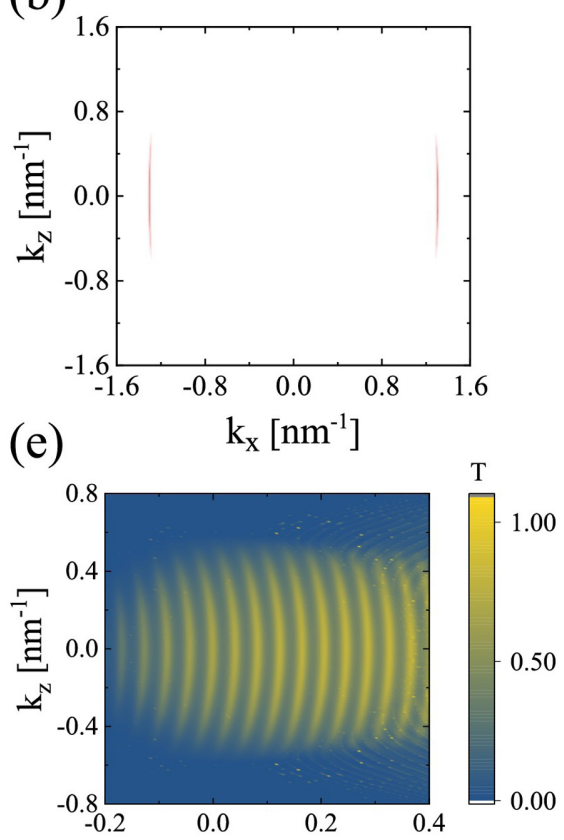

(h)

$\varepsilon[\mathrm{eV}]$

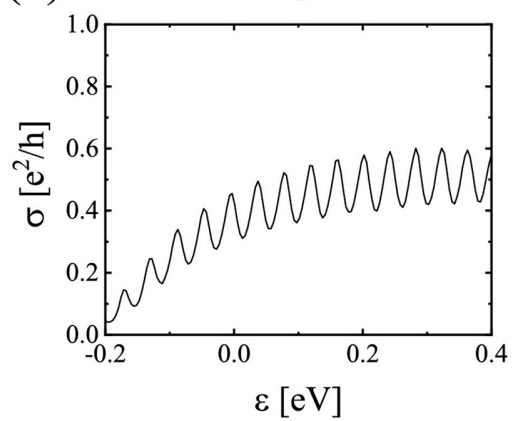

(c)
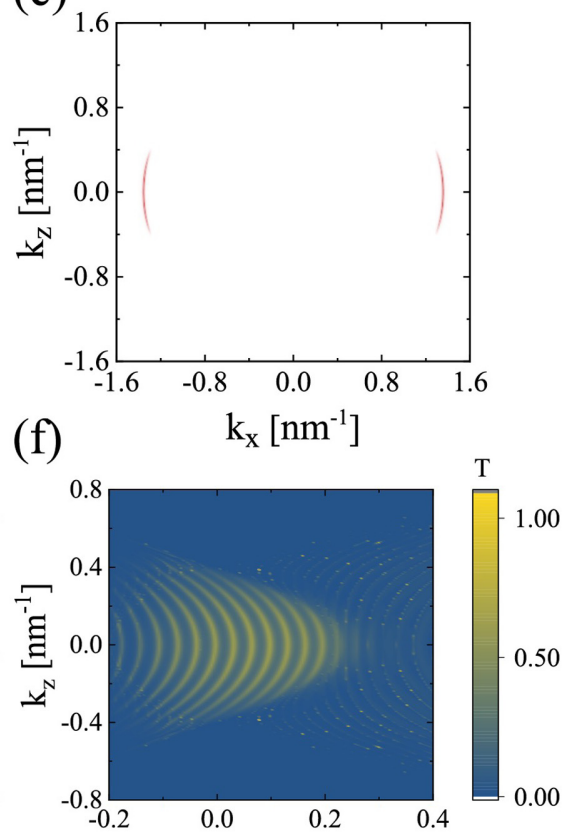

(i)

$\varepsilon[\mathrm{eV}]$

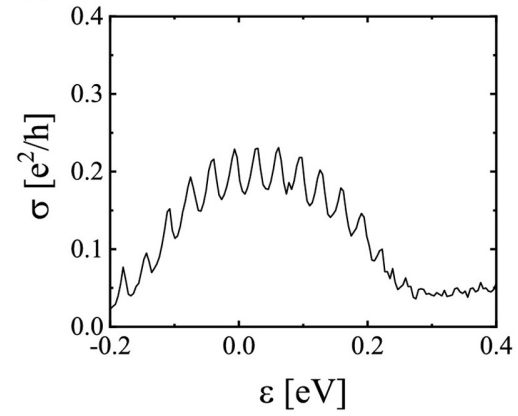

FIG. 3. FAs and conductance oscillations for different WSM given by Eq. (13), with $V$ being the on-site potential on the surface layer. Top rows illustrate the FA spectra with different curvature and length, where the relevant parameters are (a) $k_{0}=0.6 \mathrm{~nm}^{-1}, V=1.0 \mathrm{eV}$; (b) $k_{0}=0.6 \mathrm{~nm}^{-1}, V=0.2 \mathrm{eV}$; and (c) $k_{0}=0.4 \mathrm{~nm}^{-1}, V=1.0 \mathrm{eV}$. [(d)-(f)] Middle rows illustrate the corresponding numerical results of the transmission coefficient $T$ as a function of $\varepsilon$ and $k_{z}$. [(g)-(i)] Bottom rows exhibit the corresponding conductance $\sigma$ after integrates over $k_{z}$. The other parameters are $a=1 \mathrm{~nm}, U_{1}=U_{2}=1.0 \mathrm{eV}, M_{1}=M_{2}=1.25 \mathrm{eV} \mathrm{nm}^{2}, v_{y}=0.66 \mathrm{eV} \mathrm{nm}, k_{1}=1.2 \mathrm{~nm}^{-1}, C=0.5 \mathrm{eV} \mathrm{nm}^{2}$, $\mu_{N}=1.0 \mathrm{eV}$, and $t_{N}=0.5 \mathrm{eV} \mathrm{nm}^{2}$.

the bright stripes in the transmission pattern in Figs. 3(d)-3(f). These results are in coincidence with the analytical calculations in Fig. 2. Although the shape of the FAs is difficult to change for specific samples, the above results provide a guidance for choosing proper materials of the WSM to realize the proposed phenomenon.

Since disorder and impurity are usually present in real systems, next we investigate the effect of disorder on the conductance oscillations. We add an uncorrelated Gaussian disorder potential in the region of the WSM. The transmission pattern and the resultant conductance are shown in Fig. 4. One can see that the periodicity of the conductance oscillation maintains up to the disorder strength of $0.1 \mathrm{eV}$. As it increases further, i.e., $0.2 \mathrm{eV}$, the conductance spectrum becomes irregular.

In the above calculations, for simplicity, we set the chemical potential to zero in the WSM to have a vanishing density of the bulk states. To simulate a finite density of states in real materials, we set the Fermi surface of the WSM to be at $0.2 \mathrm{eV}$. The transmission pattern and the resultant conductance in this case are shown in Figs. 5(a) and 5(b). One can see that the conductance spectrum still exhibits a distinct oscillation [Figs. 5(b)]. To explicitly show the contribution of the bulk states, we plot the band structures and the corresponding spatial current distributions in Figs. 5(c)-5(h) for various $k_{z}$ slices and a finite Fermi energy. One can see that the electrons reaching the right electrode mostly flow along the surface [Figs. 5(f) and 5(g)]. For a finite Fermi energy, the main effect due to the bulk states is certain leakage of the injected electrons into the bulk [Figs. 5(h)]. However, the dissipated electrons are hardly regained by the other surface electrode thus having negligible contribution to the surface transport [Fig. 5(h)]. As a result, the surface states dominate the local density of surface states so as to dominate the surface transport as well. 
(a)

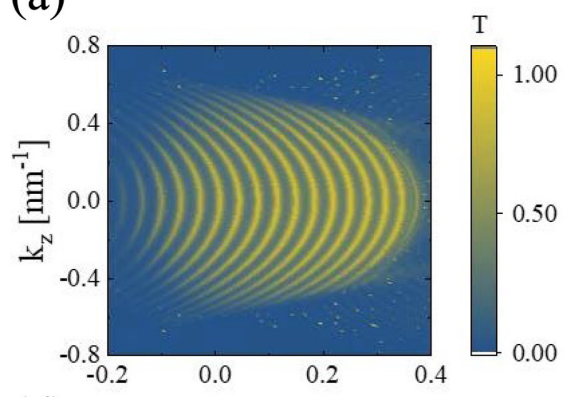

(d)

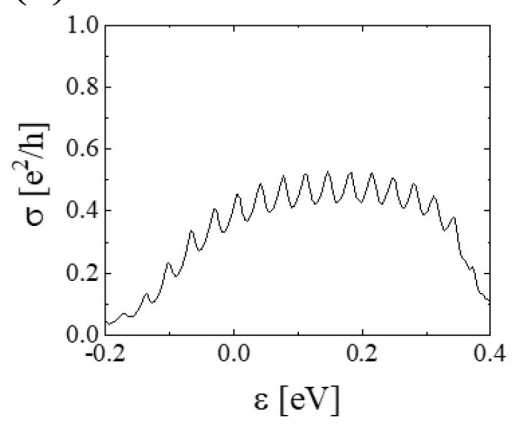

(b)

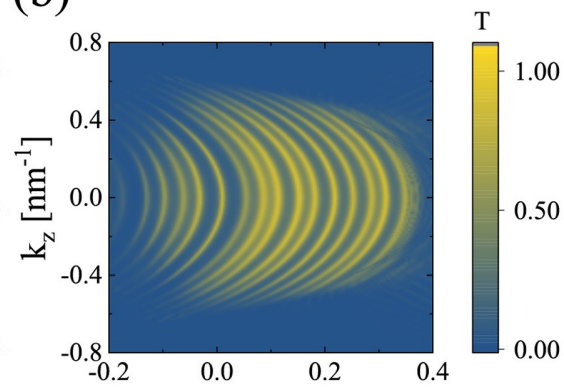

(e)

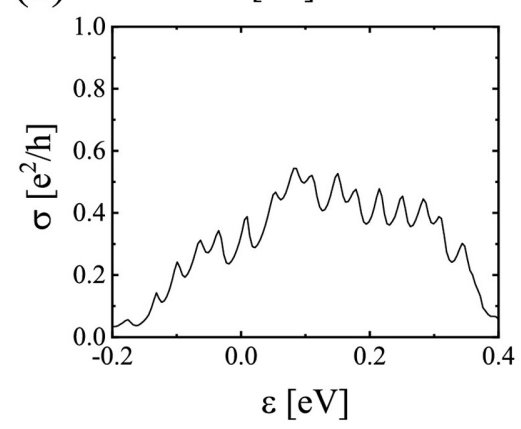

(c)

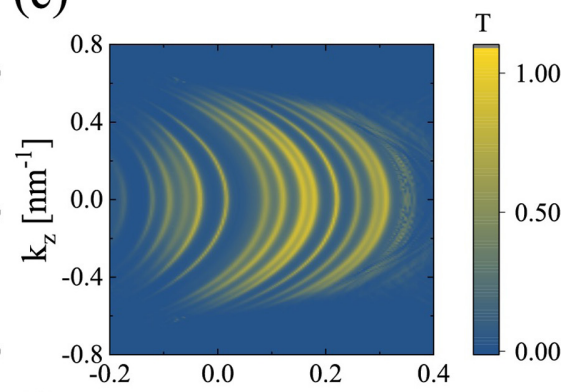

(f)

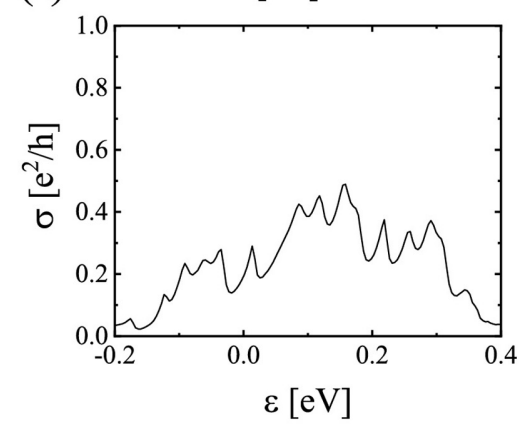

FIG. 4. Conductance oscillations in disordered WSMs. Top rows illustrate the transmission coefficient $T$ as a function of $\varepsilon$ and $k_{z}$, where the disorder strengths are (a) 0 , (b) 0.1 , and (c) $0.2 \mathrm{eV}$. Bottom rows exhibit the corresponding conductance $\sigma$ after integrates over $k_{z}$. Other parameters are the same as those in Fig. $3(\mathrm{~g})$.

\section{ORIENTATION DEPENDENT CONDUCTANCE SPECTRA}

In the above section, we have seen that the shape of the FAs strongly affect the oscillation pattern of the conductance. In addition, FAs can also have diverse orientations relative to the normal of the strip electrodes with $\theta \neq 0$. Experimentally, this can be achieved by fabricating the strip electrodes along intended directions and theoretically, this can be described by the effective model $H_{W}(\boldsymbol{k}, \theta)$ in Eq. (4) with a finite rotation while keeping the normal of the electrodes fixed to the $\hat{x}$ direction.

The numerical calculation is performed on the discretized version of Hamiltonian $H_{W}(\boldsymbol{k}, \theta)$ in the same way as that in the previous section. Again, the momentum $k_{z}$ is taken as a parameter. In Figs. 6(a)-6(c), we plot the FAs with different azimuthal angles $\theta$. One can see that the rotation of the effective Hamiltonian causes corresponding rotated FAs. The transmission probabilities $T\left(k_{z}, \varepsilon\right)$ as a function of energy $\varepsilon$ and $k_{z}$ are shown in Figs. 6(d)-6(f). One can see that there in general exist two distinct regions in the transmission pattern as shown in Fig. 6(e) except for two limiting cases, $\theta=0$ in Fig. 6(d) and $\theta \geqslant \tan ^{-1}\left(k_{0} / k_{1}\right)$ 6(f). Specifically, the regions with stripe structures and nearly uniform strength correspond to regions I and II in Fig. 1(b), respectively. In region I, backscattering channels are available which induces interference and oscillation of the transmission, while in region II, the transport channels are chiral with a high transmission without oscillation. The amounts of $k_{z}$ channels in region I and II vary with $\theta$, which is clearly revealed in the conductance spectra in Figs. 6(g)-6(i). As $\theta$ increases from zero, the oscillation of conductance becomes less visible, because the ratio between the numbers of $k_{z}$ channels in regions I and II becomes smaller. When $\theta$ exceeds the threshold $\tan ^{-1}\left(k_{0} / k_{1}\right)$ [Fig. 6(c)], all electrons reside in region II and the conductance exhibits a plateau structure without any oscillation as shown in Fig. 6(i). Such a transition from oscillation to plateau structure in the conductance spectra provides a clear manifestation of the highly anisotropic nature of the FAs, and therefore can serve as its unique signal. Although we elucidate such an effect based on a specific model in Eq. (4), the underlying physics should generally hold for other WSMs with more complicated FA configurations. The shape of FAs and the relative amount of $k_{z}$ channels lying in regions I and II are of most importance for the main results.

\section{MAGNETIC FIELD EFFECT}

In this section, we show that the visibility of the conductance oscillation can be well improved by a magnetic field in the $\hat{y}$ direction. We focus on the case $\theta=0$ and the results are shown in Fig. 7. The Landau gauge $\boldsymbol{A}=(0,0,-B x)$ is adopted such that the Peierls substitution $\boldsymbol{k} \rightarrow-i \boldsymbol{\nabla}-\boldsymbol{e A} / \hbar$ (with $e>0$ ) retains the $k_{z}$ conservation. For a small magnetic field which satisfies $B \ll \hbar k_{0,1} /(e a)$, it only introduces a smooth modification of the mass term in Eq. (1). Such a pseudo-spin-dependent potential contributes an additional phase factor in the transmission function $T\left(k_{z}, \varepsilon, B\right)$, which causes a shift of the pattern in the $k_{z}$ direction, as can be seen in Figs. 7(d)-7(f). A constant gauge term $\delta A$ can always be added to the vector potential as $\boldsymbol{A}^{\prime}=(0,0,-B x+\delta A)$, which is equivalent to an overall shift of $k_{z}$. The physical results of the conductance spectra should not rely on such freedom of gauge choice by noting that the Hamiltonian is a periodic function of $k_{z}$, so that an overall shift of $k_{z}$ by $\delta A$ has no 

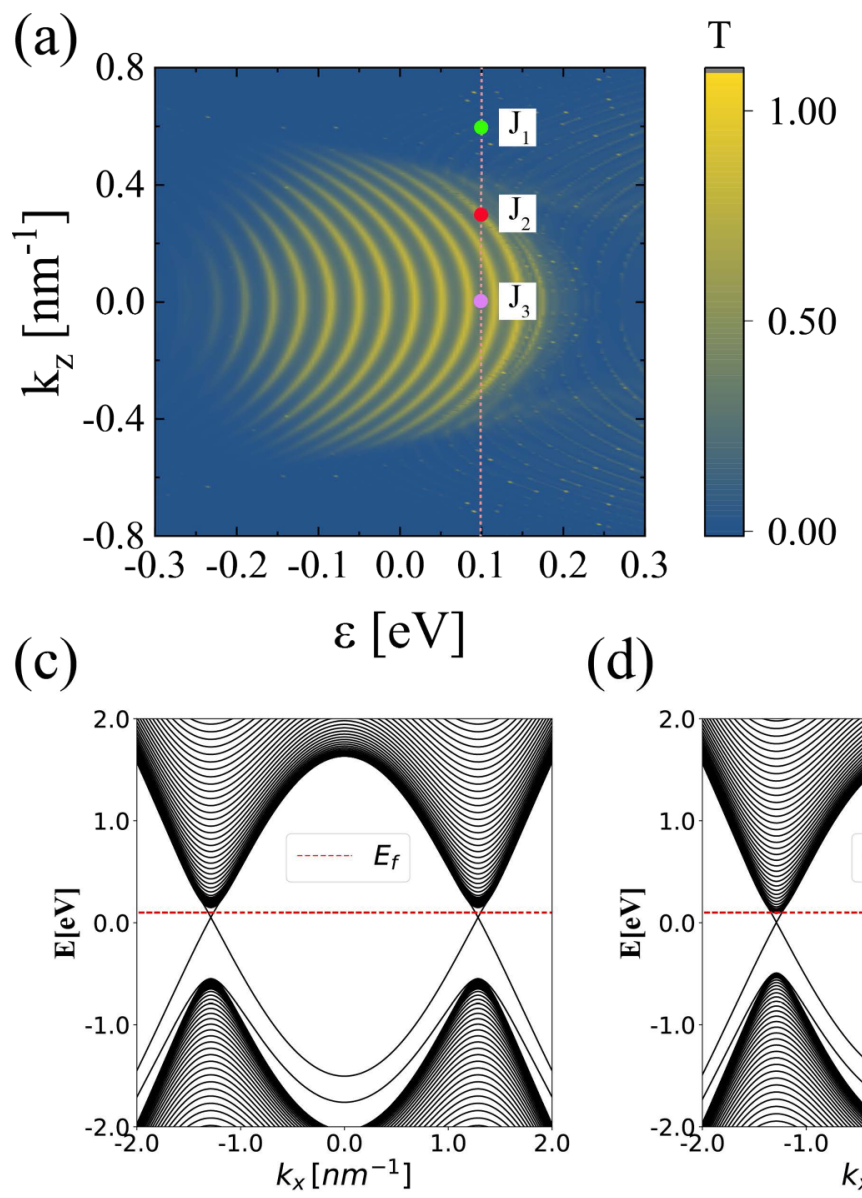

(d)

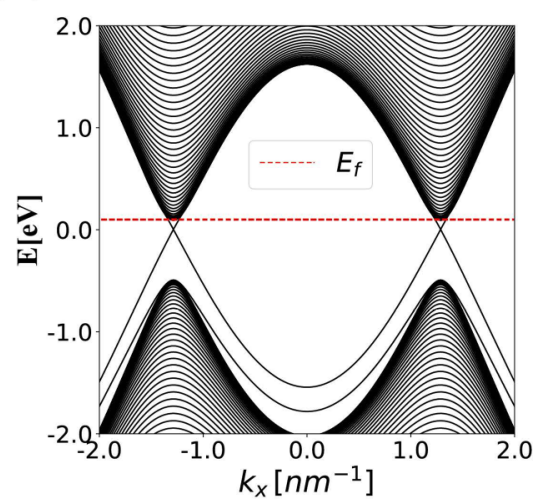

(e)
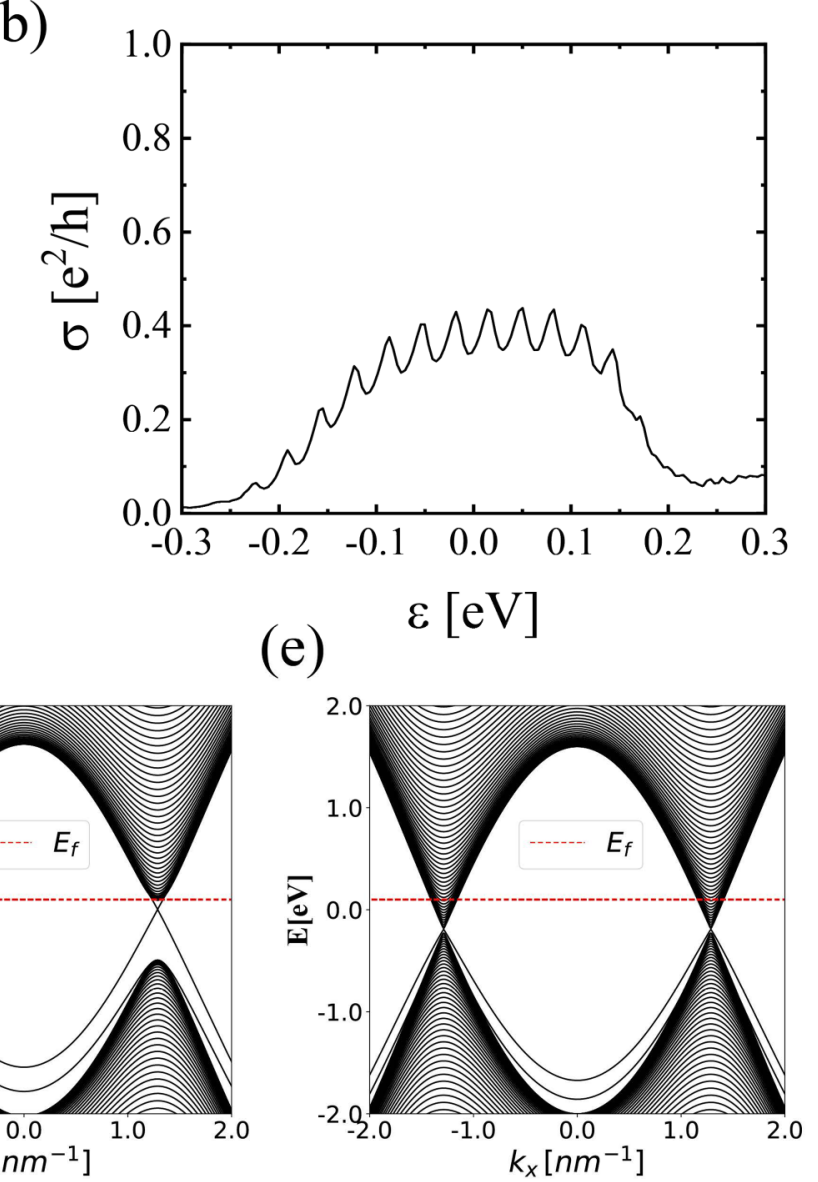

(f)
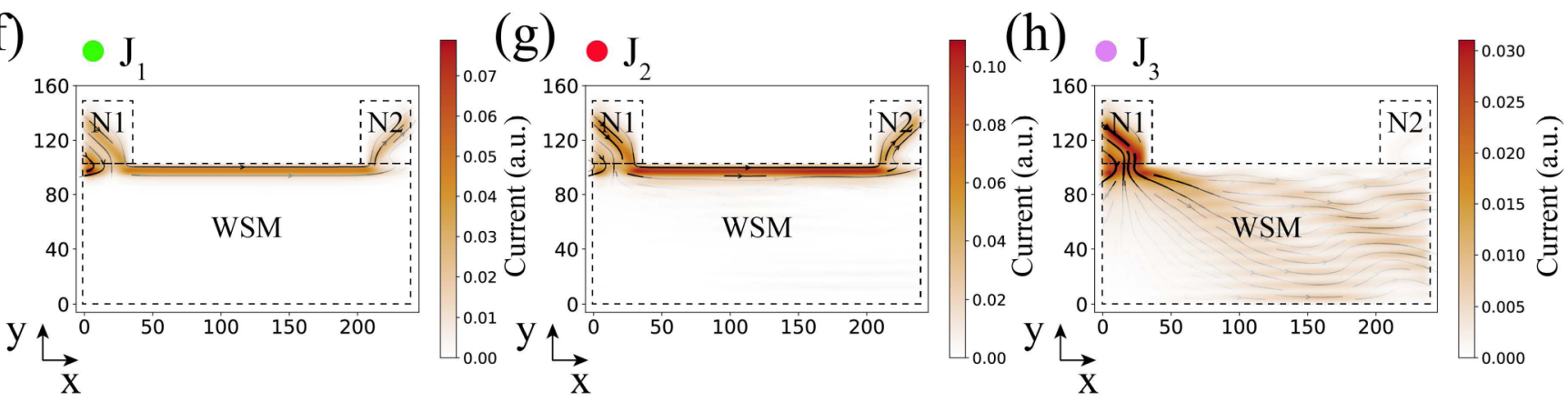

FIG. 5. (a) The transmission coefficient $T$ as a function of $\varepsilon$ and $k_{z}$ when the Fermi surface of the WSM is set to $0.2 \mathrm{eV}$. (b) The corresponding differential conductance after summing over the transverse channels $k_{z}$. The band structures of the $k_{z}$ channels with (c) $k_{z}=0$ nm ${ }^{-1}$, (d) $k_{z}=0.3 \mathrm{~nm}^{-1}$, and (e) $k_{z}=0.6 \mathrm{~nm}^{-1}$. The spatial current distributions (f) $J_{1}$, (g) $J_{2}$, and (h) $J_{3}$ for various $k_{z}$ slice and a finite Fermi energy $0.1 \mathrm{eV}$ marked in panel (a). The other parameters are the same as those in Fig. 3(g).

effect after integration. The magnetic field effect can also be well understood by the semiclassical picture of Lorentz force. The Lorentz force drives electrons sliding along the FAs [Fig. 7(a)], which corresponds to the curved trajectory in real space [Fig. 7(b)].

Remarkably, because that FAs are terminated at the Weyl points, some of the electrons nearby can transfer into the chiral Landau bands of the bulk states and dissipate due to the surface-bulk connection at the Weyl points [65], as illustrated by the dashed green circles in Fig. 7(a). As a result, these electrons cannot reach the right electrode and do not contribute to the conductance. Therefore, the magnetic field effectively reduces the number of $k_{z}$ channels and thus the dephasing effect, which is reflected in Figs. 7(d)-7(f) that the interference patterns get narrower in the $k_{z}$ direction as $B$ increases. Accordingly, one can see in Figs. 7(g)-7(i) that as $B$ increases, the magnitude of the conductance reduces due to the loss of surface electrons. However, the oscillation of the conductance becomes more visible stemming from weaker dephasing. To visualize the transport in the NFAN junctions, we plot the current distributions for a given $k_{z}$ and $\varepsilon$ marked in Fig. 7(e) as shown in Fig. 8. One can see a clear connection between the bulk and the surface states in Fig. 8(a), which is the real-space manifestation of the Weyl orbit. Due to the magnetic field, these electrons cannot return back to the electrodes, so that the conductance is reduced. Meanwhile, the electrons away 
(a)

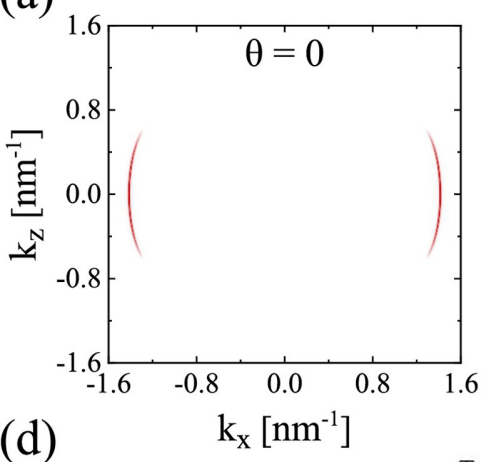

(d)

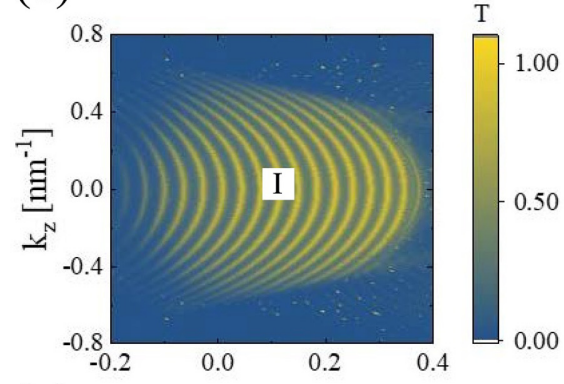

(g)

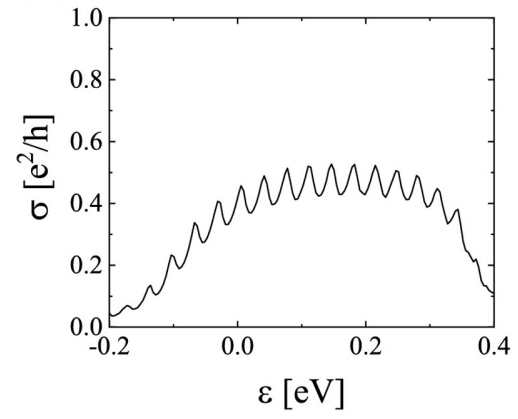

(b)
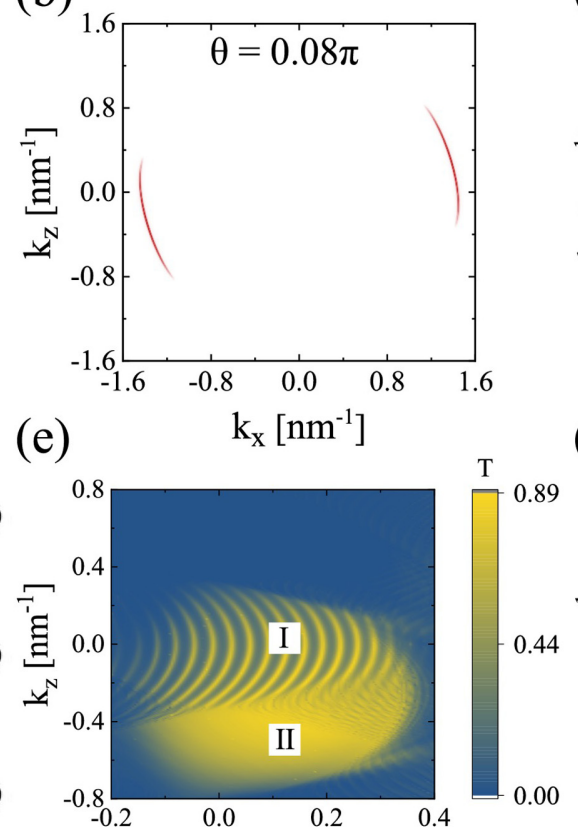

(h)

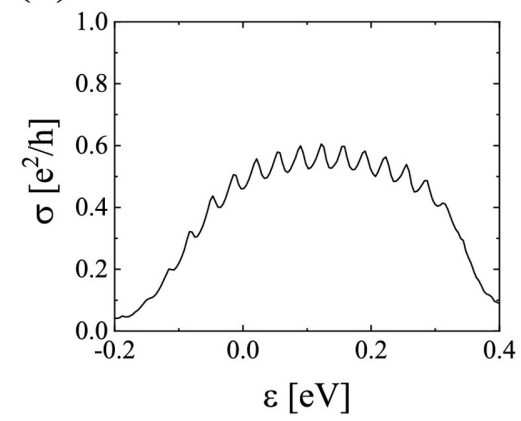

(c)
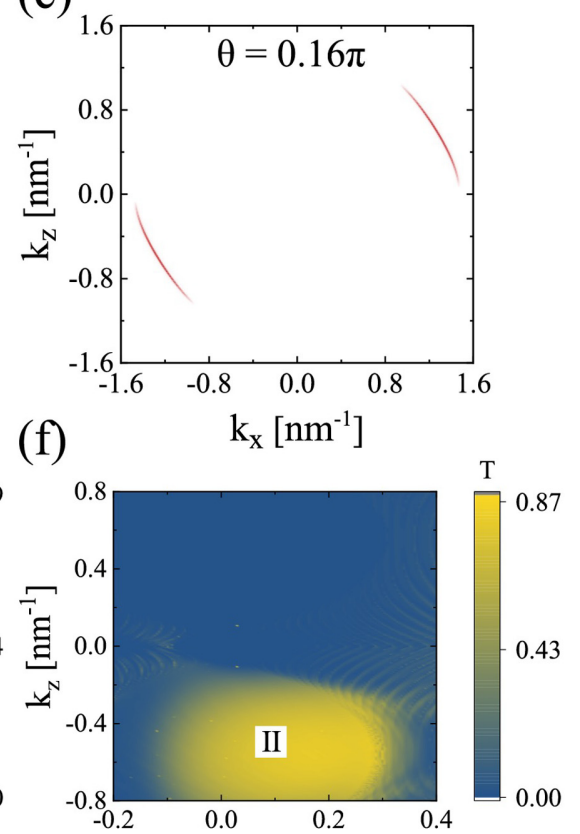

(i)

$\varepsilon[\mathrm{eV}]$

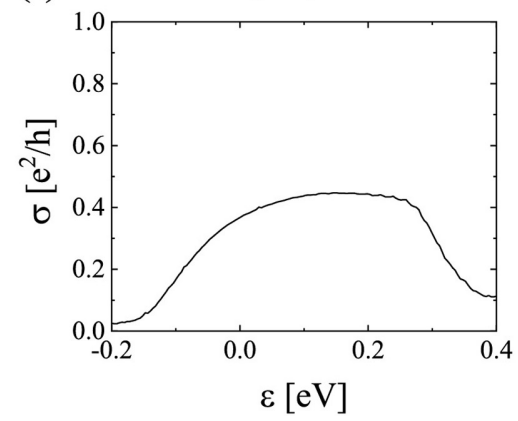

FIG. 6. Conductance oscillations in WSM with FAs of different orientations. [(a)-(c)] Upper panel: FA spectra for different azimuthal angles $\theta$. [(d)-(f)] Middle panel: Corresponding transmission probability as a function of $\varepsilon$ and $k_{z}$. [(g)-(i)] Lower panel: Corresponding differential conductance after summing over all the transverse channels $k_{z}$. The other parameters are the same as those in Fig. 3(g).

from the Weyl point can still flow along the top surface and reach the electrode on the right side [cf. Fig. 8(b)].

To quantify the visibility of conductance oscillation, we introduce the resolution defined as follows,

$$
R=\sum_{i}^{8} \frac{\sigma_{i}^{\max }-\sigma_{i}^{\min }}{\sigma_{i}^{\max }+\sigma_{i}^{\min }},
$$

where $\sigma_{i}^{\max }$ and $\sigma_{i}^{\min }$ are the neighboring maximum and minimum values of the conductance. We choose the most visible eight oscillating periods to calculate the resolution and plot $R$ as a function of $B$ in Fig. 7(c). We see that the resolution increases significantly with increasing magnetic field, indicating that the observation of 2D Fabry-Pérot interference can be facilitated by applying a magnetic field, in stark contrast to other 2D electronic systems [53-59]. Such a novel effect can also be used as a direct evidence of FAs.

We remark that there exists a critical magnetic field $B_{c}=$ $\hbar K_{z} /(e L)$ in the calculation, above which all the incident electrons in the FA surface states will transfer into the bulk and no surface transport occurs. Here, $K_{z}$ is the span of the FA in the $k_{z}$ direction [Fig. 7(a)] and $L$ is the distance between the two electrodes [Fig. 1(a)]. For parameters $K_{z}=1.2 \mathrm{~nm}^{-1}$ and $L=180 \mathrm{~nm}$ adopted in Fig. 7, we have $B_{c} \simeq 4.4$ Tesla.

\section{DISCUSSION AND SUMMARY}

We would like to discuss the experimental realization of our proposal. The surface NFAN junctions can be achieved by state-of-the-art fabrication techniques [66-68]. In the calculations, we have assumed the specular reflection at the contacts. In general, the diffusive boundary condition will introduce the coupling between different transverse $k_{z}$ slices, which leads to the dephasing effect. Therefore, high-quality contacts are required for the observation of the conductance oscillation. Good contacts with smooth boundaries can be implemented by state-of-the-art fabrication techniques [66], like electronbeam lithography $[69,70]$. For example, the length of the electrodes along the smooth boundary can reach $4 \mu \mathrm{m}$ [66], whereas the Fermi wavelength of the WSM in our system is $\frac{2 \pi}{k_{1}}=5.24 \mathrm{~nm}$. The size of the electrodes along the boundary is much larger than the Fermi wavelength and if their 
(a)

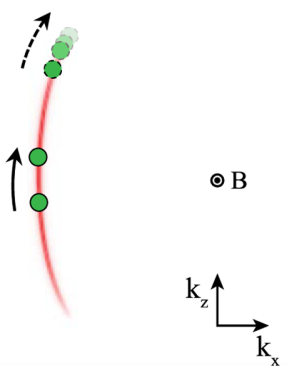

(d)

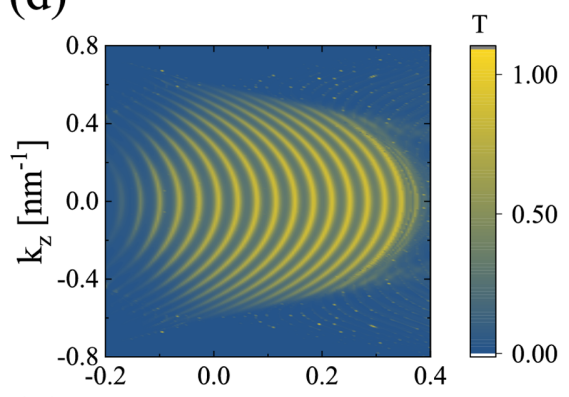

(g)
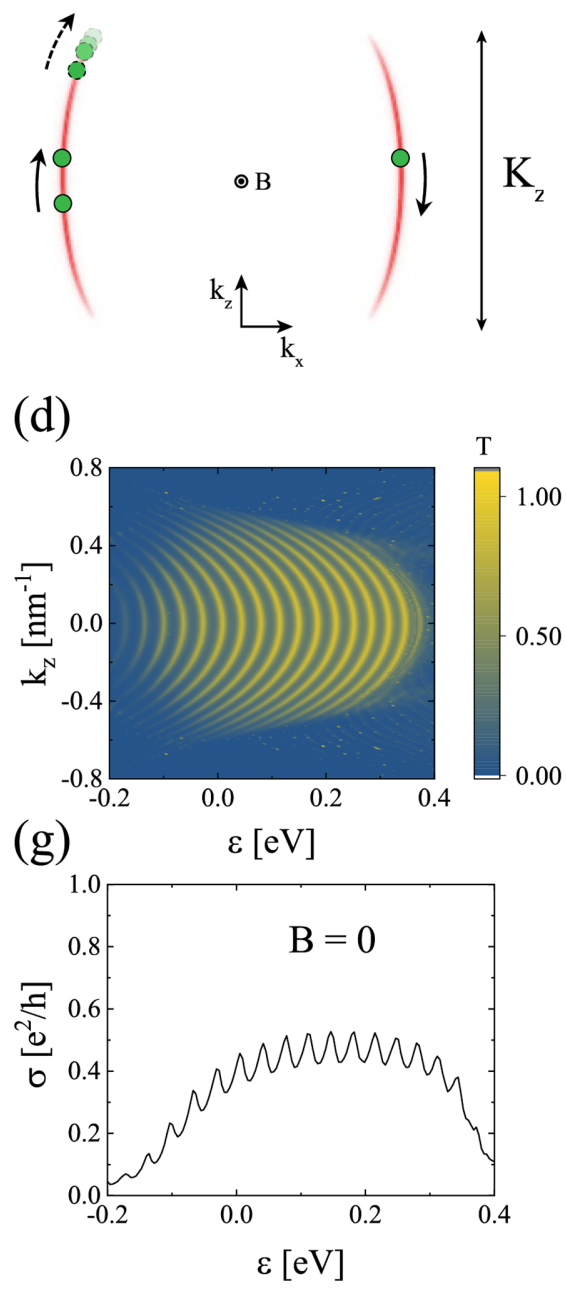

(b)

(e)
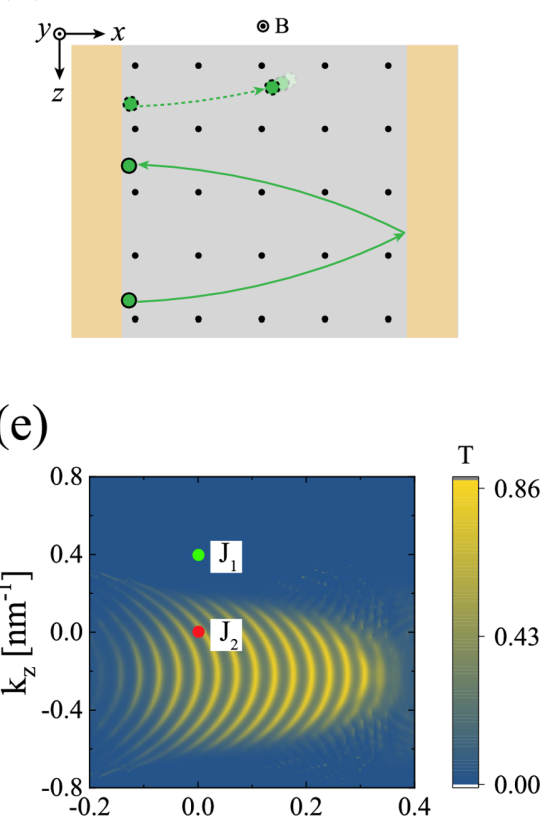

(h)

$\varepsilon[\mathrm{eV}]$

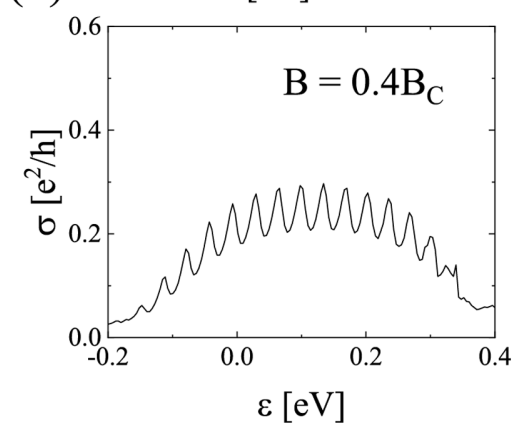

(c)

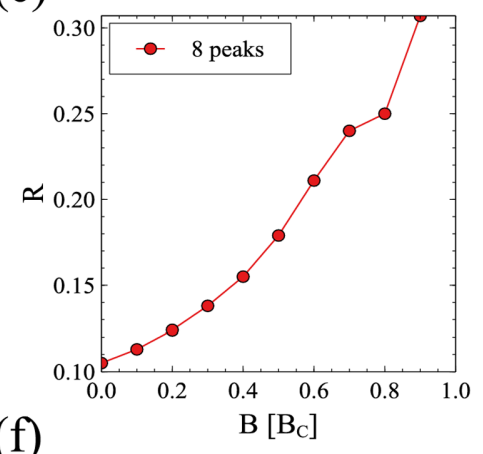

(f)

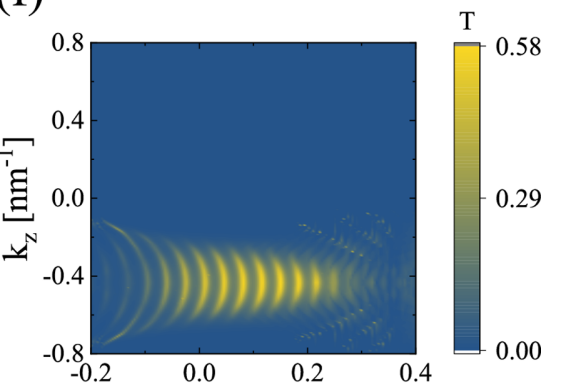

(i)

$\varepsilon[\mathrm{eV}]$

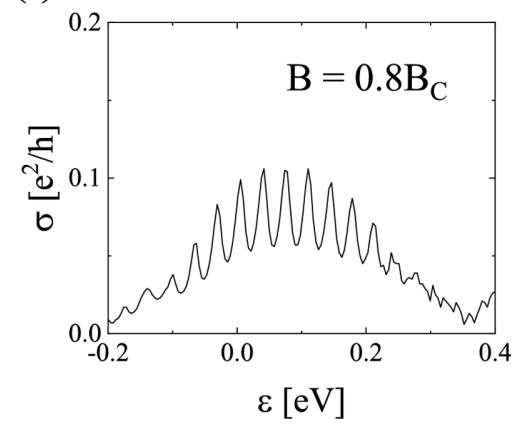

FIG. 7. Conductance oscillations in a WSM under a magnetic field perpendicular to the surface. (a) FA spectrum for azimuthal angle $\theta=0$, and electrons slide along the FA driven by the Lorentz force. (b) Trajectories of electrons in real space corresponding to the left panel. (c) Resolution of the conductance as a function of the magnetic field, where $B_{C}$ is the saturated magnetic field. The transmission probability as a function of $\varepsilon$ and $k_{z}$ with (d) $B=0$, (e) $B=0.4 B_{C}$, and (f) $B=0.8 B_{C}$. [(g)-(i)] The corresponding conductance after summing over all the transverse channels $k_{z}$ for different magnetic fields in the $\hat{y}$ direction. The other parameters are the same for those in Fig. 3(g).

(a)

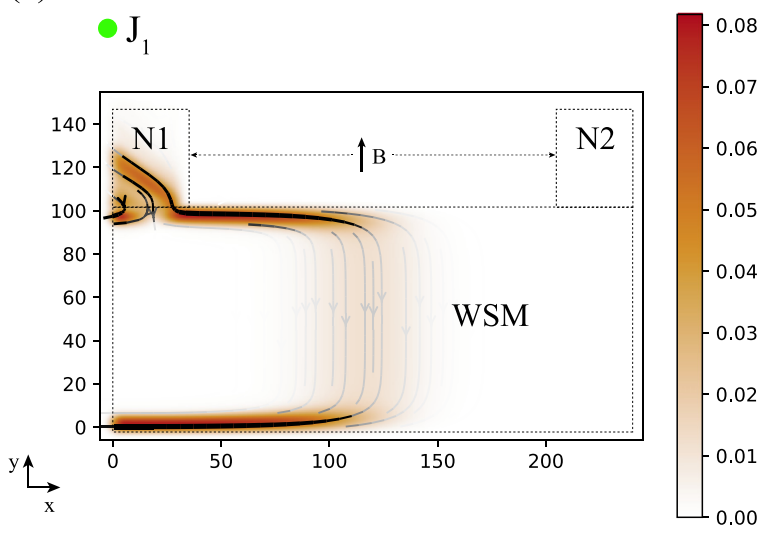

(b)

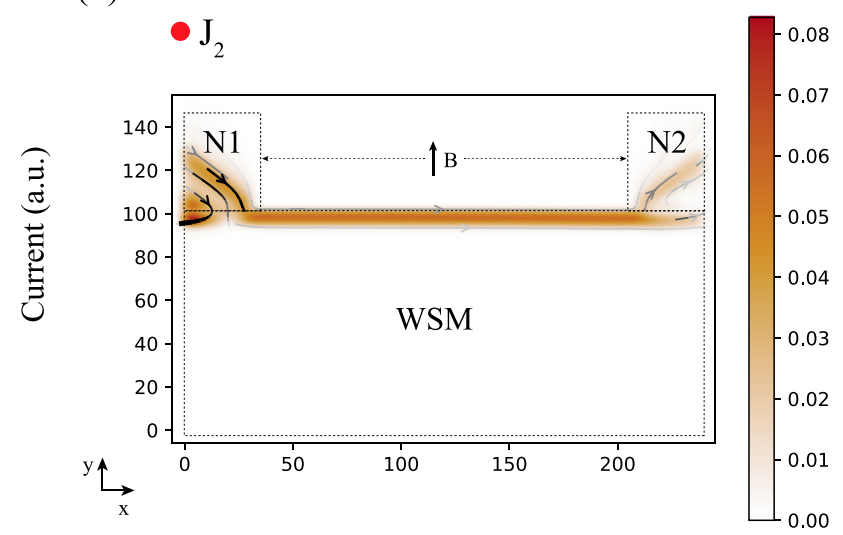

FIG. 8. The current distributions (a) $J_{1}$ and (b) $J_{2}$ for a given $k_{z}$ and $\varepsilon$ marked in Fig. 7(e). 
boundaries are smooth enough, then the transverse momentum $k_{z}$ is approximately conserved during scattering. The WSM with a pair of FAs is a crucial building block in our proposal, which has been reported in $\mathrm{NbIrTe}_{4}$ [49,61-63], $\mathrm{WP}_{2}$ [71], $\mathrm{MoTe}_{2}$ [72], and $\mathrm{YbMnBi}_{2}$ [73]. The main reason for the choice of the current time-reversal $(\mathcal{T})$-symmetric model is that it is the minimal model which contains a pair of FAs. Here, the number of Fermi arcs is important, but not the timereversal symmetry. The main results still hold for other materials with more FAs as long as the two regions of transmission can be well defined. During the calculation, for simplicity, we set the chemical potential to zero in the WSM to have a vanishing density of the bulk states. In real materials with finite density of states, our main conclusions remain unchanged as long as the FAs and the bulk states are well separated in the surface Brillouin zone. The presence of bulk states will only cause certain leakage of surface electrons, but will not change the present qualitative results, as discussed in Fig. 5.

To summarize, we have investigated the 2D conductance oscillation in the planar NFAN junctions on the WSM surface, which provides a unique transport signature of the FAs. It is found that (i) shorter and less curved FAs can lead to more visible conductance oscillation, and the conductance oscillations are robust to weak disorder and a slight shift of the Fermi surface; (ii) a crossover from oscillation to plateau structure of the conductance spectra can be implemented by changing the orientation of the planar junctions; and (iii) the magnetic field can significantly enhance the visibility of the oscillation pattern which is unique for the FA surface states. Therefore, our work offers an effective way to identify FA surface states through transport measurement. It also introduces a new platform to realize interesting $2 \mathrm{D}$ conductance oscillation induced by Fabry-Pérot-type interference.

\section{ACKNOWLEDGMENTS}

This work was supported by the National Natural Science Foundation of China under Grant No. 12074172 (W.C.), the startup grant at Nanjing University (W.C.), the State Key Program for Basic Researches of China under Grant No. 2017YFA0303203 (D.Y.X.), and the Excellent Programme at Nanjing University.
[1] H. Weyl, Proc. Nat. Acad. Sci. USA 15, 323 (1929).

[2] N. P. Armitage, E. J. Mele, and A. Vishwanath, Rev. Mod. Phys. 90, 015001 (2018).

[3] T. Kajita, Rev. Mod. Phys. 88, 030501 (2016).

[4] A. B. McDonald, Rev. Mod. Phys. 88, 030502 (2016).

[5] X. Wan, A. M. Turner, A. Vishwanath, and S. Y. Savrasov, Phys. Rev. B 83, 205101 (2011).

[6] S. Murakami, New J. Phys. 9, 356 (2007).

[7] A. A. Burkov and L. Balents, Phys. Rev. Lett. 107, 127205 (2011).

[8] H. Weng, C. Fang, Z. Fang, B. A. Bernevig, and X. Dai, Phys. Rev. X 5, 011029 (2015).

[9] S.-M. Huang, S.-Y. Xu, I. Belopolski, C.-C. Lee, G. Chang, B. Wang, N. Alidoust, G. Bian, M. Neupane, C. Zhang et al., Nat. Commun. 6, 7373 (2015)

[10] B. Q. Lv, H. M. Weng, B. B. Fu, X. P. Wang, H. Miao, J. Ma, P. Richard, X. C. Huang, L. X. Zhao, G. F. Chen, Z. Fang, X. Dai, T. Qian, and H. Ding, Phys. Rev. X 5, 031013 (2015)

[11] S.-Y. Xu, I. Belopolski, N. Alidoust, M. Neupane, G. Bian, C. Zhang, R. Sankar, G. Chang, Z. Yuan, C.-C. Lee, S.-M. Huang, H. Zheng, J. Ma, D. S. Sanchez, B. Wang, A. Bansil, F. Chou, P. P. Shibayev, H. Lin, S. Jia, and M. Z. Hasan, Science 349, 613 (2015).

[12] S.-Y. Xu, N. Alidoust, I. Belopolski, Z. Yuan, G. Bian, T.-R. Chang, H. Zheng, V. N. Strocov, D. S. Sanchez, G. Chang et al., Nat. Phys. 11, 748 (2015).

[13] S.-Y. Xu, I. Belopolski, D. S. Sanchez, C. Zhang, G. Chang, C. Guo, G. Bian, Z. Yuan, H. Lu, T.-R. Chang, P. P. Shibayev, M. L. Prokopovych, N. Alidoust, H. Zheng, C.-C. Lee, S.-M. Huang, R. Sankar, F. Chou, C.-H. Hsu, H.-T. Jeng, A. Bansil, T. Neupert, V. N. Strocov, H. Lin, S. Jia, and M. Z. Hasan, Sci. Adv. 1, e1501092 (2015).
[14] N. Xu, H. Weng, B. Lv, C. E. Matt, J. Park, F. Bisti, V. N. Strocov, D. Gawryluk, E. Pomjakushina, K. Conder et al., Nat. Commun. 7, 11006 (2016).

[15] K. Deng, G. Wan, P. Deng, K. Zhang, S. Ding, E. Wang, M. Yan, H. Huang, H. Zhang, Z. Xu et al., Nat. Phys. 12, 1105 (2016).

[16] L. Yang, Z. Liu, Y. Sun, H. Peng, H. Yang, T. Zhang, B. Zhou, Y. Zhang, Y. Guo, M. Rahn et al., Nat. Phys. 11, 728 (2015).

[17] L. Huang, T. M. McCormick, M. Ochi, Z. Zhao, M.-T. Suzuki, R. Arita, Y. Wu, D. Mou, H. Cao, J. Yan et al., Nat. Mater. 15, 1155 (2016).

[18] A. Tamai, Q. S. Wu, I. Cucchi, F. Y. Bruno, S. Riccò, T. K. Kim, M. Hoesch, C. Barreteau, E. Giannini, C. Besnard, A. A. Soluyanov, and F. Baumberger, Phys. Rev. X 6, 031021 (2016).

[19] J. Jiang, Z. Liu, Y. Sun, H. Yang, C. Rajamathi, Y. Qi, L. Yang, C. Chen, H. Peng, C. Hwang et al., Nat. Commun. 8, 13973 (2017).

[20] I. Belopolski, D. S. Sanchez, Y. Ishida, X. Pan, P. Yu, S.-Y. $\mathrm{Xu}, \mathrm{G}$. Chang, T.-R. Chang, H. Zheng, N. Alidoust et al., Nat. Commun. 7, 13643 (2016).

[21] B. Lv, N. Xu, H. Weng, J. Ma, P. Richard, X. Huang, L. Zhao, G. Chen, C. Matt, F. Bisti et al., Nat. Phys. 11, 724 (2015).

[22] A. A. Zyuzin and A. A. Burkov, Phys. Rev. B 86, 115133 (2012).

[23] V. Aji, Phys. Rev. B 85, 241101(R) (2012).

[24] D. T. Son and B. Z. Spivak, Phys. Rev. B 88, 104412 (2013).

[25] M. N. Chernodub, A. Cortijo, A. G. Grushin, K. Landsteiner, and M. A. H. Vozmediano, Phys. Rev. B 89, 081407(R) (2014).

[26] J.-H. Zhou, H. Jiang, Q. Niu, and J.-R. Shi, Chin. Phys. Lett. 30, 027101 (2013).

[27] A. A. Burkov, J. Phys.: Condens. Matter 27, 113201 (2015). 
[28] J. Ma and D. A. Pesin, Phys. Rev. B 92, 235205 (2015).

[29] S. Zhong, J. E. Moore, and I. Souza, Phys. Rev. Lett. 116, 077201 (2016).

[30] B. Z. Spivak and A. V. Andreev, Phys. Rev. B 93, 085107 (2016).

[31] M. Hirschberger, S. Kushwaha, Z. Wang, Q. Gibson, S. Liang, C. A. Belvin, B. A. Bernevig, R. J. Cava, and N. P. Ong, Nat. Mater. 15, 1161 (2016).

[32] X. Huang, L. Zhao, Y. Long, P. Wang, D. Chen, Z. Yang, H. Liang, M. Xue, H. Weng, Z. Fang, X. Dai, and G. Chen, Phys. Rev. X 5, 031023 (2015).

[33] C. Shekhar, A. K. Nayak, Y. Sun, M. Schmidt, M. Nicklas, I. Leermakers, U. Zeitler, Y. Skourski, J. Wosnitza, Z. Liu et al., Nat. Phys. 11, 645 (2015).

[34] J. Du, H. Wang, Q. Chen, Q. Mao, R. Khan, B. Xu, Y. Zhou, Y. Zhang, J. Yang, B. Chen et al., Sci. China Phys. Mech. Astron. 59, 657406 (2016).

[35] Z. Wang, Y. Zheng, Z. Shen, Y. Lu, H. Fang, F. Sheng, Y. Zhou, X. Yang, Y. Li, C. Feng, and Z.-A. Xu, Phys. Rev. B 93, 121112(R) (2016).

[36] C.-L. Zhang, S.-Y. Xu, I. Belopolski, Z. Yuan, Z. Lin, B. Tong, G. Bian, N. Alidoust, C.-C. Lee, S.-M. Huang et al., Nat. Commun. 7, 10735 (2016).

[37] H. Nielsen and M. Ninomiya, Nucl. Phys. B 185, 20 (1981).

[38] H. Nielsen and M. Ninomiya, Nucl. Phys. B 193, 173 (1981).

[39] H. Nielsen and M. Ninomiya, Phys. Lett. B 130, 389 (1983).

[40] E. V. Gorbar, V. A. Miransky, I. A. Shovkovy, and P. O. Sukhachov, Phys. Rev. B 93, 235127 (2016).

[41] V. Kaladzhyan and J. H. Bardarson, Phys. Rev. B 100, 085424 (2019).

[42] P. O. Sukhachov, M. V. Rakov, O. M. Teslyk, and E. V. Gorbar, Ann. Phys. 532, 1900449 (2020).

[43] S. Pinon, V. Kaladzhyan, and C. Bena, Phys. Rev. B 101, 115405 (2020).

[44] G. Murthy, H. A. Fertig, and E. Shimshoni, Phys. Rev. Res. 2, 013367 (2020).

[45] S. Kourtis, J. Li, Z. Wang, A. Yazdani, and B. A. Bernevig, Phys. Rev. B 93, 041109(R) (2016).

[46] S. Pinon, V. Kaladzhyan, and C. Bena, Phys. Rev. B 102, 165117 (2020).

[47] N. Morali, R. Batabyal, P. K. Nag, E. Liu, Q. Xu, Y. Sun, B. Yan, C. Felser, N. Avraham, and H. Beidenkopf, Science $\mathbf{3 6 5}$, 1286 (2019).

[48] H. Yang, L. Yang, Z. Liu, Y. Sun, C. Chen, H. Peng, M. Schmidt, D. Prabhakaran, B. A. Bernevig, C. Felser et al., Nat. Commun. 10, 3478 (2019).

[49] S. A. Ekahana, Y. W. Li, Y. Sun, H. Namiki, H. F. Yang, J. Jiang, L. X. Yang, W. J. Shi, C. F. Zhang, D. Pei, C. Chen, T. Sasagawa, C. Felser, B. H. Yan, Z. K. Liu, and Y. L. Chen, Phys. Rev. B 102, 085126 (2020).

[50] W. Chen, K. Luo, L. Li, and O. Zilberberg, Phys. Rev. Lett. 121, 166802 (2018).

[51] G. Chen, O. Zilberberg, and W. Chen, Phys. Rev. B 101, 125407 (2020).

[52] Y. Zheng, W. Chen, and D. Y. Xing, Phys. Rev. B 104, 075420 (2021).
[53] A. F. Young and P. Kim, Nat. Phys. 5, 222 (2008).

[54] P. Rickhaus, R. Maurand, M. H. Liu, M. Weiss, K. Richter, and C. Schönenberger, Nat. Commun. 4, 2342 (2013).

[55] A. L. Grushina, D.-K. Ki, and A. F. Morpurgo, Appl. Phys. Lett. 102, 223102 (2013).

[56] M. Oksanen, A. Uppstu, A. Laitinen, D. J. Cox, M. F. Craciun, S. Russo, A. Harju, and P. Hakonen, Phys. Rev. B 89, 121414(R) (2014).

[57] L. C. Campos, A. F. Young, K. Surakitbovorn, K. Watanabe, T. Taniguchi, and P. Jarillo-Herrero, Nat. Commun. 3, 1239 (2012).

[58] A. Varlet, M.-H. Liu, V. Krueckl, D. Bischoff, P. Simonet, K. Watanabe, T. Taniguchi, K. Richter, K. Ensslin, and T. Ihn, Phys. Rev. Lett. 113, 116601 (2014).

[59] M. Karalic, A. Štrkalj, M. Masseroni, W. Chen, C. Mittag, T. Tschirky, W. Wegscheider, T. Ihn, K. Ensslin, and O. Zilberberg, Phys. Rev. X 10, 031007 (2020).

[60] S. Datta, Electronic Transport in Mesoscopic Systems (Cambridge University Press, Cambridge, UK, 1997).

[61] K. Koepernik, D. Kasinathan, D. V. Efremov, S. Khim, S. Borisenko, B. Büchner, and J. van den Brink, Phys. Rev. B 93, 201101(R) (2016)

[62] I. Belopolski, P. Yu, D. S. Sanchez, Y. Ishida, T.-R. Chang, S. S. Zhang, S.-Y. Xu, H. Zheng, G. Chang, G. Bian et al., Nat. Commun. 8, 942 (2017).

[63] E. Haubold, K. Koepernik, D. Efremov, S. Khim, A. Fedorov, Y. Kushnirenko, J. van den Brink, S. Wurmehl, B. Büchner, T. K. Kim, M. Hoesch, K. Sumida, K. Taguchi, T. Yoshikawa, A. Kimura, T. Okuda, and S. V. Borisenko, Phys. Rev. B 95, 241108(R) (2017).

[64] C. W. Groth, M. Wimmer, A. R. Akhmerov, and X. Waintal, New J. Phys. 16, 063065 (2014).

[65] A. C. Potter, I. Kimchi, and A. Vishwanath, Nat. Commun. 5, 5161 (2014).

[66] C.-Z. Li, A.-Q. Wang, C. Li, W.-Z. Zheng, A. Brinkman, D.-P. Yu, and Z.-M. Liao, Nat. Commun. 11, 1150 (2020).

[67] A. Q. Chen, M. J. Park, S. T. Gill, Y. Xiao, D. R.-i. Plessis, G. J. MacDougall, M. J. Gilbert, and N. Mason, Nat. Commun. 9, 3478 (2018).

[68] S. Ghatak, O. Breunig, F. Yang, Z. Wang, A. A. Taskin, and Y. Ando, Nano Lett. 18, 5124 (2018).

[69] C. Déprez, L. Veyrat, H. Vignaud, G. Nayak, K. Watanabe, T. Taniguchi, F. Gay, H. Sellier, and B. Sacépé, Nat. Nanotechnol. 16, 555 (2021).

[70] Y. Ronen, T. Werkmeister, D. H. Najafabadi, A. T. Pierce, L. E. Anderson, Y. J. Shin, S. Y. Lee, Y. H. Lee, B. Johnson, K. Watanabe et al., Nat. Nanotechnol. 16, 563 (2021).

[71] M.-Y. Yao, N. Xu, Q. S. Wu, G. Autès, N. Kumar, V. N. Strocov, N. C. Plumb, M. Radovic, O. V. Yazyev, C. Felser, J. Mesot, and M. Shi, Phys. Rev. Lett. 122, 176402 (2019).

[72] Z. Wang, D. Gresch, A. A. Soluyanov, W. Xie, S. Kushwaha, X. Dai, M. Troyer, R. J. Cava, and B. A. Bernevig, Phys. Rev. Lett. 117, 056805 (2016).

[73] S. Borisenko, D. Evtushinsky, Q. Gibson, A. Yaresko, K. Koepernik, T. Kim, M. Ali, J. van den Brink, M. Hoesch, A. Fedorov et al., Nat. Commun. 10, 3424 (2019). 